\title{
Identificación de flavonoles en un extracto polar de Aciotis polystachya (Bonpland) Triana (Melastomataceae)
}

\author{
Identification of flavonols in polar extract in Aciotis polystachya \\ (Bonpland) Triana (Melastomataceae)
}

\author{
Manuel Haminton Salas-Moreno*
}

\begin{abstract}
Resumen
Objetivo: Aislar, identificar y elucidar los metabolitos secundarios presentes en algunas fracciones del extracto acetato de etilo de las partes aéreas de Aciotis polystachya. Metodología: Mediante las técnicas de cromatografía de columna, capa fina y preparativa, se aislaron, identificaron y purificaron los metabolitos secundarios; el análisis de los espectros de $\mathrm{RMN}-{ }^{1} \mathrm{H}, \mathrm{RMN}-{ }^{13} \mathrm{C}$ y técnicas bidimensionales de RMN ( ${ }^{1} \mathrm{H}-{ }^{1} \mathrm{H}$, COSY y HMQC) permitió elucidar la estructura química de estos metabolitos. Resultados: Al extracto metanólico de Aciotis polystachya se le realizó una extracción sólido a líquido con acetato de etilo; de las fracciones 57-64 de la cromatografía general del extracto acetato de etilo, eluida con los solventes diclorometano-acetato de etilo en proporciones volumétricas de 40-60\%, se aislaron 0,03 gramos de un sólido de color amarillo después de sucesivas cromatografías, de igual forma las fracciones 65-73 y 57-64 provenientes de la misma cromatografía; se obtuvieron en total 0,13 gramos de producto en forma de cristales amarillos. Conclusiones: La Quercetina y el Kaemferol constituyen un nuevo reporte de metabolitos secundarios tanto para el género como para esta especie, además son los responsables de muchas actividades biológicas o farmacológicas, lo cual podría justificar algunos usos etnobotánicos de esta planta.
\end{abstract}

Palabras clave: Aciotis polystachya, Flavonoles, Melastomataceae, Kaempferol, Quercetina, RMN.

\section{Abstract}

Objetive: To isolate, to identify and to elucidate the secondary metabolites present in the same fraction extract ethyl acetate of air parts of Aciotis polystachya (Bonplan) Triana (Melastomataceae). Methodology: Techniques using column chromatography, thin layer chromatography and preparative chromatography was isolate, identify and purify secondary metabolites, the analysis of the spectra of ${ }^{1} \mathrm{H}-N M R,{ }^{13} \mathrm{C}-N M R$ and two-dimensional NMR techniques $\left({ }^{1} \mathrm{H}-{ }^{1} \mathrm{H}\right.$ COSY and $\left.\mathrm{HMQC}\right)$ allowed us to elucidate the chemical structure of these metabolites. Results: The methanol extract was performed Aciotis polystachya solid to liquid extraction with ethyl acetate, fractions 57-64 from the chromatography of the extract usually ethyl acetate, eluted with solvent dichloromethane-ethyl acetate in volumetric proportions $40-60 \%$, 0.03 grams was isolated as a yellow solid after successive chromatographies, similarly fractions 65-73 and 57-64 from the same chromatography was obtained 0.13 grams of total product as yellow crystals. Conclusions: Quercetin and Kaempherol are new secondary metabolites report both gender as this species, and are responsible for many biological and pharmacological activities, which justify some ethnobotanical uses of this plant.

Keywords: Aciotis polystachya, Flavonols, Melastomataceae, Kaemphferol, Quercetin, NMR.

\section{Introducción}

Aciotis polistachya es una hierba de 15 a $50 \mathrm{~cm}$ de altura, erecta con tallos cuadrangulares, con abun- dantes tricomas glandulares, rosados o blanquecinos. Hojas cordiformes u ovadas, margen serrulada, verde oscuro, con tricomas glandulares rojizos o blanquecinos, inflorescencia encima doble, con flores que

Universidad Tecnológica del Chocó, Facultad de Ciencias Naturales, Grupo de Investigación en Biosistemática, línea de Investigación en Fitoquímica de Melastomatáceas, Quibdó, Chocó, Colombia. e-mail: hasamo49@ hotmail.com 
poseen pétalos, con tricomas glandulares. Estambres con filamentos, anteras púrpuras; el fruto es una cápsula (Cuesta-Sánchez y Palacios-Duque 2007). En Perú se utiliza para tratar la tuberculosis y dolencias en los riñones; se distribuyen principalmente en Colombia, Ecuador, Perú, Venezuela y en la Amazonia brasileña (Freire-Fierro 2002). Son muy escasos los estudios a nivel químicos o de actividad biológica en esta especie, sin embargo Salas-Moreno (2011) aisló y elucidó a partir del extracto diclorometánico de las partes aéreas de $A$. polistachya las saponinas esteroidales la 3-O- $\beta$-D-glucopiranósido de estigmasterol y la 3-O- $\beta$-D-glucopiranósido de $\beta$ sitosterol. En A. purpuracens se evaluó la actividad inhibitoria de xantina oxidasa de los extractos isopropanol-agua, aunque los porcentajes de inhibición no superaron el 10\% en las proporciones (1:1 y 30:70); en proporciones 30:70 alcanzó 20\%. De igual manera Isaza et al. (2007) evaluaron la actividad alelopática de algunas especies de Melastomataceas entre ellas Aciotis. En general las plantas de la familia Melastomataceae se han usado por años en la medicina tradicional, sobre todo en Asia y América Latina como astringentes o hemostáticos; en casos de diarrea, disentería, leucorrea y enfermedades de la piel (Perry y Metzger 1980), contra afecciones respiratorias (Renner 1990), malaria, cálculos en la vejiga y otras enfermedades del tracto genitourinario; irritaciones en las encías y como diurético (García 1992). Entre las más de 4.500 especies de plantas Melastomataceas, agrupadas en nueve tribus (Clausing y Renner 2001), solo se han estudiado, desde el punto de vista fitoquímico, unas pocas pertenecientes a las tribus Melastomeae (Yoshida et al. 2005) y Miconieae (Rodrigues et al. 2007. Los principales metabolitos secundarios reportados para estas especies son de tipo fenólico como flavonoides glicosilados (Bomfim-Patricio et al. 2001; Isaza et al. 2001; Li et al. 2001; Zhang et al. 2003; Adriano et al. 2006).

\section{Metodología}

Recolección, secado y preparación del material vegetal. Se recolectaron las partes aéreas de la planta a partir de ejemplares adultos de A. polystachya (Figura 1), ubicados frente a la Estación Ambiental del Instituto de Investigaciones del Pacífico (IIAP), a dos horas del Municipio de Tadó, Chocó, Colombia. El material fue descrito taxonómicamente, luego secado por dos semanas a la sombra. Posteriormente se limpió y se procedió a pesarlo y molerlo en un molino manual (marca Corona).

Extracción del material vegetal. Las partes aéreas de ejemplares adultos de A. polystachya (Bonpland) Triana (Figura 1), el material vegetal secado y molido fue extraído con hexano a temperatura ambiente, durante 72 horas, secadas 24 horas bajo campana y extraídas de nuevo en dos oportunidades más con diclorometano $\left(\mathrm{CH}_{2} \mathrm{Cl}_{2}\right)$ y metanol $\left(\mathrm{CH}_{3} \mathrm{OH}\right)$ caliente en un soxhlet hasta agotamiento durante otras 24 horas. Después de obtenidos los extractos, se concentraron al vacío por medio de un rota-vapor, a una temperatura no mayor de $40^{\circ} \mathrm{C}$. En esta investigación solo se trabajó con los extractos en $\mathrm{CH}_{2} \mathrm{Cl}_{2}$ y $\mathrm{CH}_{3} \mathrm{OH}$. El extracto en hexano $\left(\mathrm{C}_{6} \mathrm{H}_{14}\right)$ solo se utilizó para depurar el material vegetal de grasas y ceras. Del extracto de $\mathrm{CH}_{2} \mathrm{Cl}_{2}$ se obtuvieron 15,6 g a partir de $1000 \mathrm{~g}$ de material vegetal. Al extracto metanólico seco, se le realizó una extracción sólido-líquido con acetato de etilo (EtOAc). El extracto de EtOAc fue rotavaporado y concentrado para obtener $6,17 \mathrm{~g}$ de extracto.

Separación y purificación de los productos. La separación se realizó utilizando la técnica de cromatografía de columna con silica gel como fase estacionaria. Como disolventes se utilizaron $\mathrm{C}_{6} \mathrm{H}_{14}$, $\mathrm{CH}_{2} \mathrm{Cl}_{2}$, EtOAc, $\mathrm{CH}_{3} \mathrm{OH}$ y mezclas de los mismos en diferentes proporciones $(1: 1 ; 1: 2 ; 1: 3$ y $100 \%)$ y de acuerdo con su perfil cromatográfico por TLC se unieron las fracciones de un litro con igual composición. A fin de facilitar la separación de los componentes de las mezclas, se utilizaron diversas técnicas tales como decantación, filtración, recristalización y cromatografía preparativa (capa gruesa) y por último, se realizó de nuevo la monitorización mediante TLC para verificar la pureza de los productos obtenidos.

Para la caracterización de los flavonoles, se utilizó la cromatografía de capa fina (TLC) y cromatografía preparativa, $\mathrm{RMN}-{ }^{1} \mathrm{H}, \mathrm{RMN}-{ }^{13} \mathrm{C}$ y técnicas bidimensionales de RMN ( ${ }^{1} \mathrm{H}-{ }^{1} \mathrm{H}$, COSY y HMQC).

\section{Resultados}

Aislamiento y purificación de los productos [1] 
A. Partes aéreas

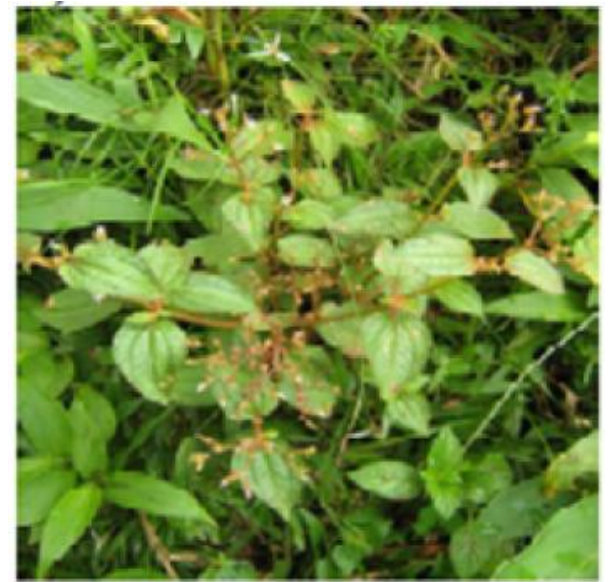

C. Flores

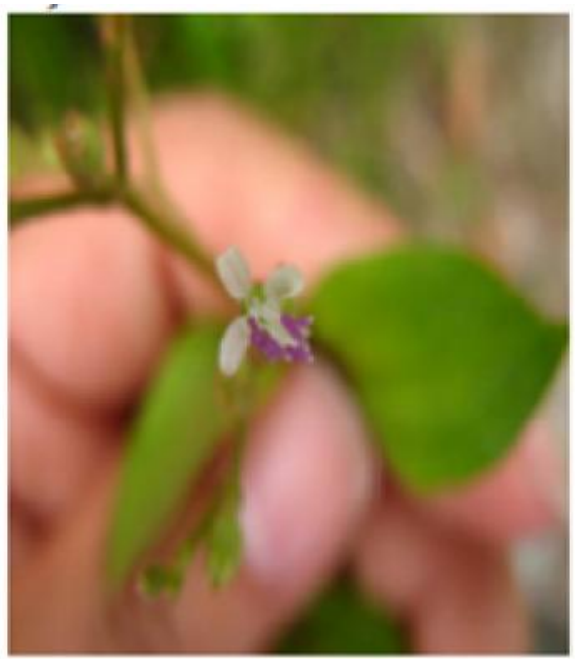

B. Frutos

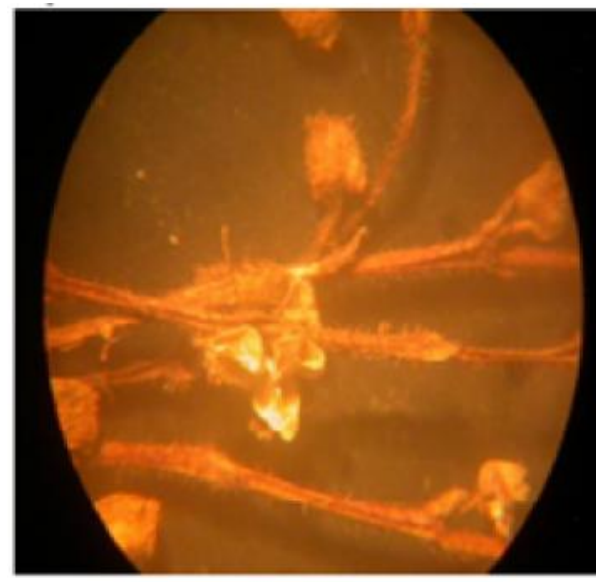

D. Inflorescencia

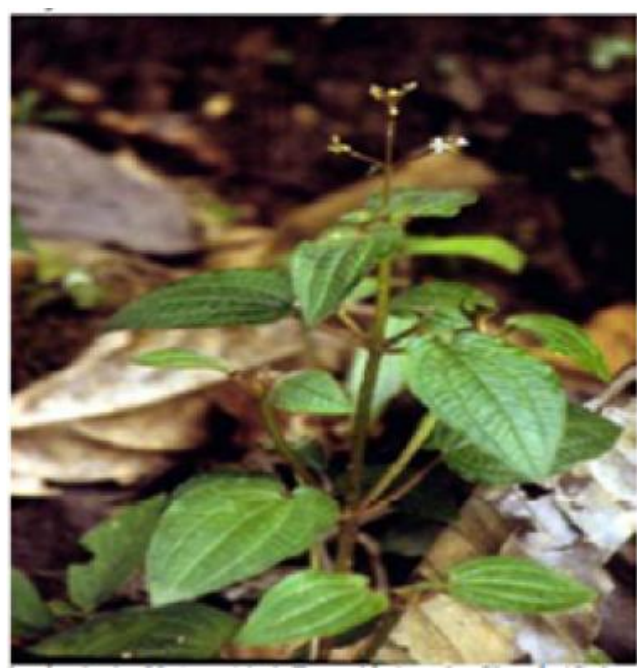

Figura 1. Aciotis polystachya (Bonpland) Triana (Melastomataceae).

$\boldsymbol{y}$ [2]. $\mathrm{Al}$ extracto $\mathrm{CH}_{3} \mathrm{OH}$ de $A$. polystachya se le realizó una extracción sólido a líquido con acetato de etilo, de las fracciones 57-64 de la cromatografía general del extracto EtOAc, eluído con los solventes $\mathrm{CH}_{2} \mathrm{Cl}_{2}$ - EtOAc en proporciones de volúmenes 40$60 \%$, se aislaron $0,03 \mathrm{~g}$ de producto como un sólido de color amarillo después de sucesivas cromatografías; presentó un punto de fusión a $269-270^{\circ} \mathrm{C}$. Fórmula nolecular: $\mathrm{C}_{15} \mathrm{H}_{10} \mathrm{O}_{6}$. Masa molecular: 286 $\mathrm{g} / \mathrm{mol}$ (Figura 2 y 3, Tabla 1 y 2). Las fracciones 65 73 y 57-64 provenientes de la cromatografía general del extracto de EtOAc en las mismas proporciones de los solventes $\mathrm{CH}_{2} \mathrm{Cl}_{2}$ - EtOAc, se obtuvieron en total $0,13 \mathrm{~g}$ de producto en forma de cristales amarillos, que presentaron un punto de fusión $316-317^{\circ} \mathrm{C}$. Fórmula molecular: $\mathrm{C}_{15} \mathrm{H}_{10} \mathrm{O}_{7}$. Masa molecular: 302 g/mol (Figura 4, Tabla 3). Para elucidar estructural- mente dichos compuestos fue necesario hacer un análisis de sus espectros de resonancia magnética nuclear unidimensional y bidimensional.

\section{Discusión}

Los desplazamiento del espectro $\mathrm{RMN}-{ }^{1} \mathrm{H}$ (Tabla 1) muestra tres señales en la zona aromática del espectro; las constantes de acoplamiento y la multiplicidad de este sistema aromático muestran que hay dos protones en posición ortorelativa ( $5^{\prime}$ y $6^{\prime}$ ) y otro meta a uno de ellos, lo que sugiere que las posiciones 3' y 4' del anillo B están sustituidas. También se observa una señal que resuena como un doblete a $6,38 \mathrm{ppm}$ y otra a $6,18 \mathrm{ppm}$, estos protones en posiciones relativas meta $(J=2,0 \mathrm{~Hz})$ muestran la presencia de dos grupos sustituyentes en las posiciones 


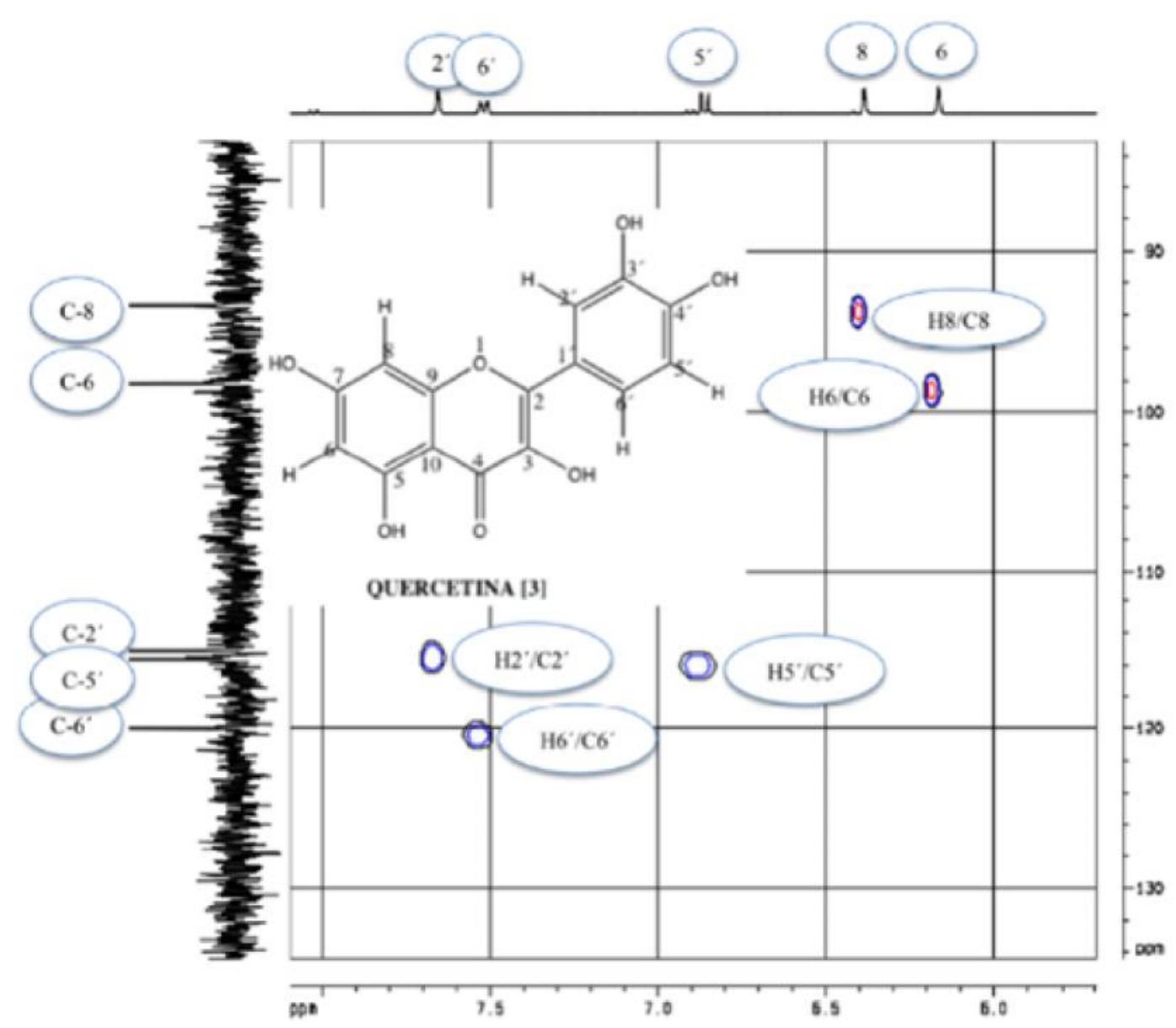

Figura 2. Espectro HMQC (DMSO- ${ }_{6}$ ) de Quercetina [1].

Tabla 1. Desplazamientos químicos $(\delta)$ en el espectro RMN-1 ${ }^{-1}$ (DMSO- ${ }_{6}, 400 \mathrm{MHz}$ ) de Quercetina [1].

\begin{tabular}{rrrrrr}
\hline $\mathrm{H}$ & $\mathrm{OH}-5$ & $\mathrm{OH}-7$ & $\mathrm{OH}-3^{\prime}$ & $\mathrm{OH}-3$ & $\mathrm{OH}-4^{\prime}$ \\
$\delta$ & 12,90 & 10,80 & 9,60 & 9,38 & 9,31 \\
$m$ & $s$ & $s$ & $s$ & $s$ & $s$ \\
$J(H z)$ & - & - & - & - & - \\
$\mathrm{H}$ & $\mathrm{H}-2^{\prime}$ & $\mathrm{H}-6^{\prime}$ & $\mathrm{H}-5^{\prime}$ & $\mathrm{H}-8$ & $\mathrm{H}-6$ \\
$\delta$ & 7,68 & 7,51 & 6,98 & 6,38 & 6,18 \\
$m$ & $d$ & $d d$ & $d$ & $d$ & $d$ \\
$J(H z)$ & 2,2 & 8,$5 ; 2,2$ & 8,5 & 2,0 & 2,0 \\
\hline
\end{tabular}

5 y 7 (además del anillo C). Los desplazamiento del espectro $\mathrm{RMN}-{ }^{13} \mathrm{C}$ (Tabla 2) se observan quince señales, de las cuales en función de sus desplazamientos químicos y orientación en el DEPT-135, cinco pertenecen a carbonos $\mathrm{sp}^{2}$ hidrogenados, una señal correspondiente a un carbonilo, insaturado $(\delta$ : $175,79 \mathrm{ppm})$ y nueve señales de carbonos $\mathrm{sp}^{2}$ cuaternarios. En el experimento HMQC (Figura 2) se observan las correlaciones de los carbonos $\mathrm{sp}^{2} \mathrm{con}$ sus respectivos protones. Además, en el experimen- to ${ }^{1} \mathrm{H},{ }^{1} \mathrm{H}$-COSY (Figura 3), se observan dos sistemas entre los protones aromáticos, un sistema $\mathrm{ABX}$, el otro sistema AX. La ausencia de grupos sustituyentes diferentes de hidroxilos, la comparación de los desplazamientos en los espectros de protónica y carbono 13 con los datos bibliográficos junto con los resultados de los diferentes experimentos espectroscópicos permitió identificar el compuesto uno como Quercetina [1].

En el espectro $\mathrm{RMN}^{1}{ }^{\mathrm{H}}$ (Tabla 3, Figura 4) del 


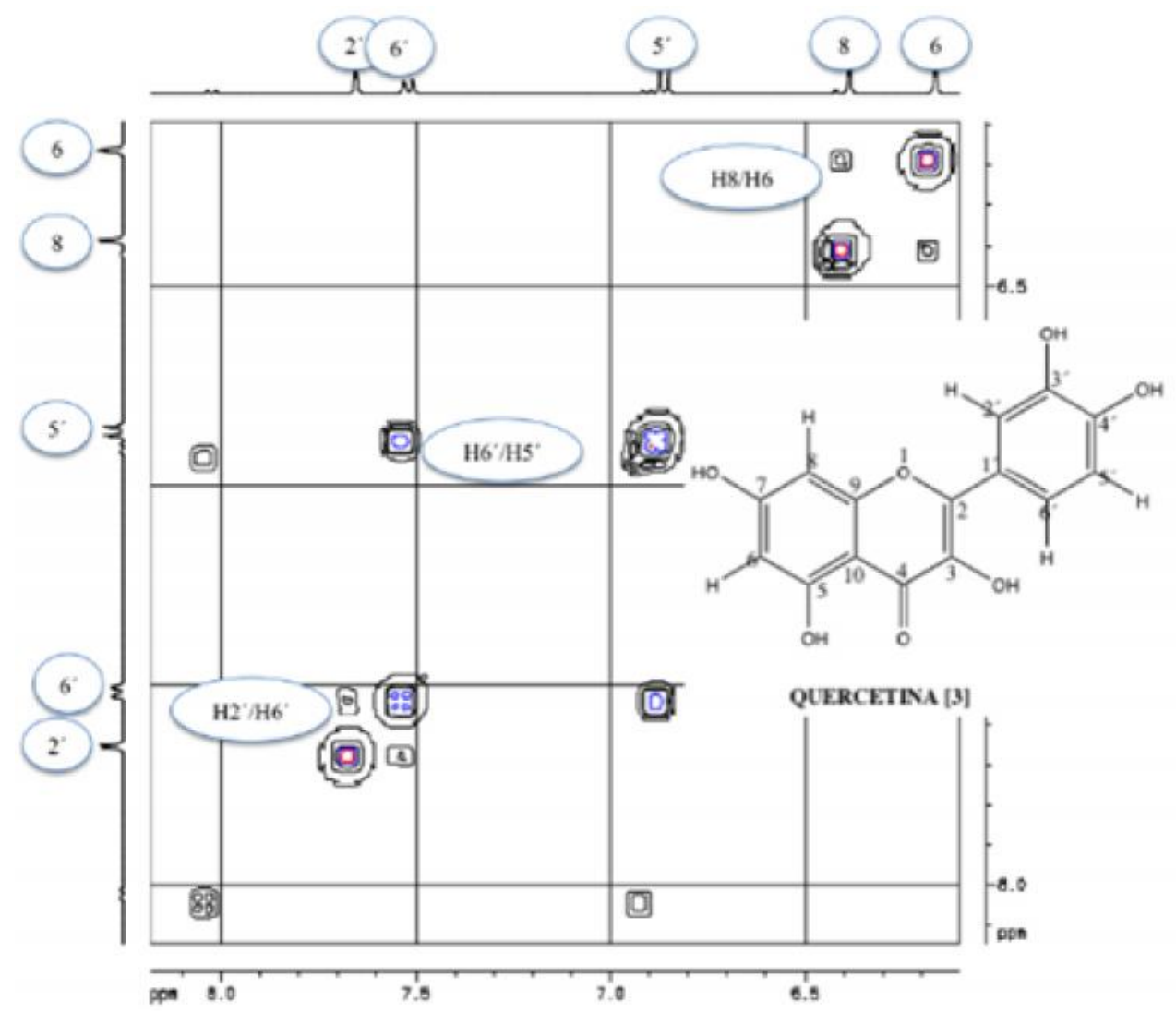

Figura 3. Espectro ${ }^{1} \mathrm{H},{ }^{1} \mathrm{H}-\mathrm{COSY}\left(\mathrm{DMSO}-\mathrm{D}_{6}\right)$ de Quercetina [1].

Tabla 2. Desplazamientos químicos $(\delta)$ en el espectro $R M N-{ }^{13} \mathrm{C}\left(\mathrm{DMSO}-\mathrm{D}_{6}, 100 \mathrm{MHz}\right)$ de Quercetina [1].

\begin{tabular}{|c|c|c|c|c|c|}
\hline C & C-2 & C-3 & C-4 & $C-5$ & C- 6 \\
\hline$\delta$ & 146,76 & 137,67 & 175,79 & 160,67 & 98,12 \\
\hline Tipo & $=\mathrm{C}-\mathrm{O}$ & $=\mathrm{C}-\mathrm{OH}$ & $>\mathrm{C}=\mathrm{O}$ & $=\mathrm{C}-\mathrm{OH}$ & $=\mathrm{C}-\mathrm{H}$ \\
\hline C & C-7 & C-8 & C-9 & C-10 & C-1' \\
\hline$\delta$ & 163,82 & 93,29 & 156,09 & 102,96 & 121,91 \\
\hline Tipo & $=\mathrm{C}-\mathrm{OH}$ & $=\mathrm{C}-\mathrm{H}$ & $=\mathrm{C}-\mathrm{O}$ & $=\mathrm{C}<$ & $=\mathrm{C}<$ \\
\hline C & C-2' & C-3' & C-4' & $C-5^{\prime}$ & C- $6^{\prime}$ \\
\hline$\delta$ & 115,02 & 145,00 & 147,75 & 115,55 & 119.92 \\
\hline Tipo & $=\mathrm{C}-\mathrm{H}$ & $=\mathrm{C}-\mathrm{OH}$ & $=\mathrm{C}-\mathrm{OH}$ & $=\mathrm{C}-\mathrm{H}$ & $=\mathrm{C}-\mathrm{H}$ \\
\hline
\end{tabular}

compuesto 2 se observan ocho señales; dos corresponden a un sistema característico de un anillo A 1,2,3,5-tetrasustituido; otras dos, que presentan dos protones equivalentes cada una, debidas al anillo $\mathrm{B}$, lo cual sugiere que la posición 4' del anillo está oxigenada; además, las otras cuatro señales a campo más bajo, todas de protones intercambiables, una de ellas a 12,48 ppm característica de los flavonoides con grupo hidroxilo en C-5. Estos desplazamientos, la multiplicidad de las señales y la cantidad de protones intercambiables, sugieren un flavonol tipo kaempferol. Las constantes de acoplamientos y la multiplicidad de estos pares de protones aromáticos equivalentes, indica que la posición $4^{\prime}$ del anillo está sustituida, lo que explica el hecho que los protones $2^{\prime}$ y $6^{\prime}$ sean equivalentes por poseer el mismo ambiente químico, de la misma forma que los protones 3'y 5', solo que los primeros se desplazan a campo más bajo porque el efecto resonante del anillo aromático los desapantalla más, porque están en posi- 
Tabla 3. Desplazamientos químicos ( $\delta$ ) en el espectro $\mathrm{RMN}^{-1} \mathrm{H}\left(\mathrm{DMSO}-\mathrm{D}_{6}, 400 \mathrm{MHz}\right.$ ) de kaempferol [2].

\begin{tabular}{|c|c|c|c|c|}
\hline $\mathrm{H}$ & $\mathrm{OH}-5$ & $\mathrm{OH}-7$ & $\mathrm{OH}-3$ & $\mathrm{OH}-4^{\prime}$ \\
\hline$\delta$ & 12,48 & 10,80 & 10,12 & 9,41 \\
\hline $\mathrm{m}$ & $\mathrm{s}$ & $\mathrm{s}$ & $\mathrm{s}$ & $\mathrm{s}$ \\
\hline$J(H z)$ & - & - & - & - \\
\hline $\mathrm{H}$ & $\mathrm{H}-2^{\prime}, 6^{\prime}$ & $\mathrm{H}-3^{\prime} ; 5^{\prime}$ & H-6 & $\mathrm{H}-8$ \\
\hline$\delta$ & 8,03 & 6,92 & 6,43 & 6,18 \\
\hline $\mathrm{m}$ & dd & dd & d & d \\
\hline$J(H z)$ & 1,$9 ; 6,9$ & $1.9 ; 6,9$ & 2,0 & 2,0 \\
\hline
\end{tabular}
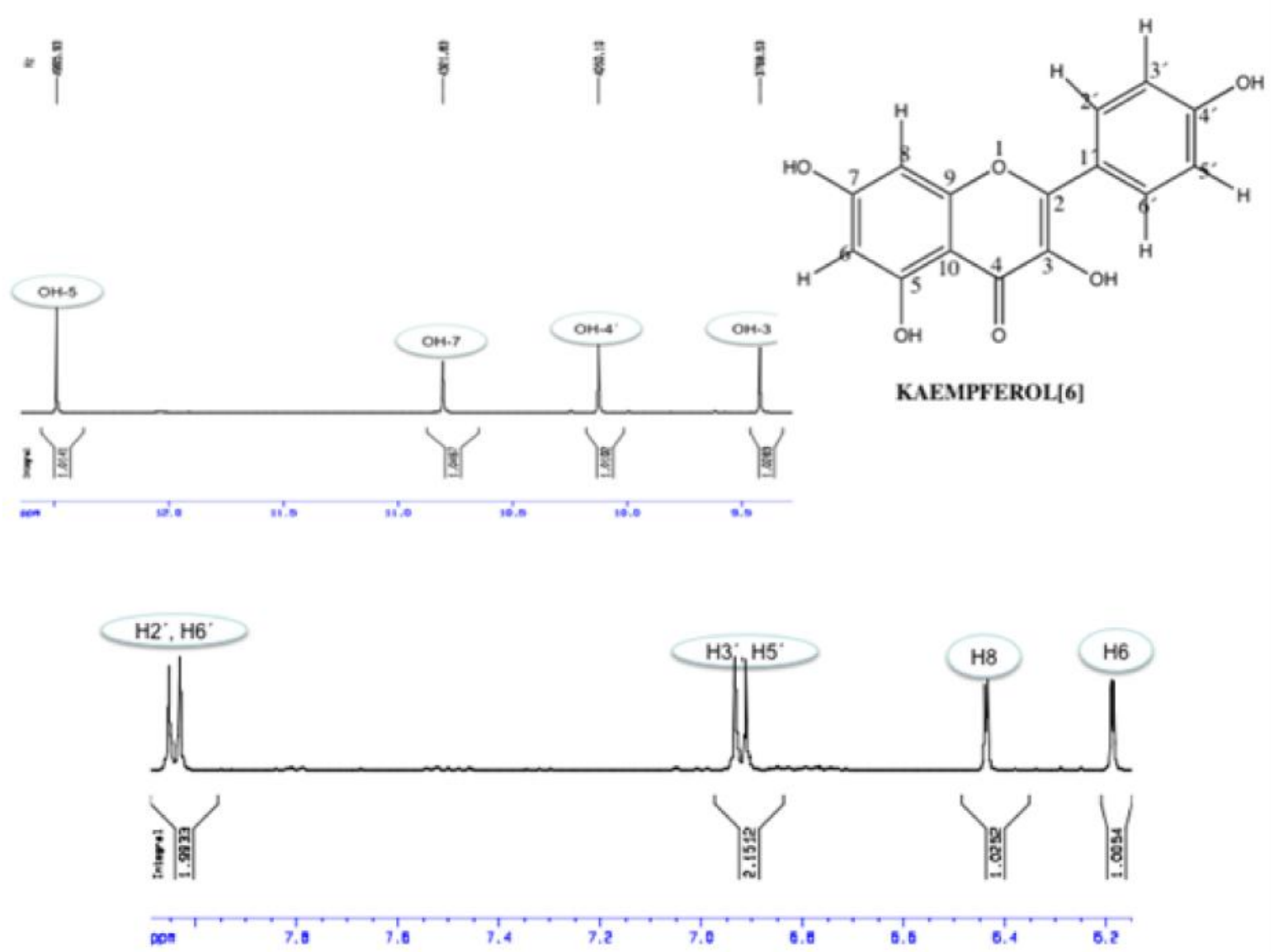

Figura 4. Espectro RMN- ${ }^{1} \mathrm{H}\left(\mathrm{DMSO}^{-\mathrm{D}_{6}}, 400 \mathrm{MHz}\right)$ de kaempferol.

ción meta con respecto al hidroxilo de la posición 4'; este sistema de protones aromáticos corresponde al anillo B del kaempferol [2]. A 6,43 ppm se observa el protón $8(1 \mathrm{H}, \mathrm{d}, J=2,0 \mathrm{~Hz})$ y a $6,18 \mathrm{ppm}$ el protón $6(1 \mathrm{H}, \mathrm{d}, J=2,0 \mathrm{~Hz})$, ambos protones conforman el anillo A del kaempferol. Estos protones en posiciones meta relativa $(J=2,0 \mathrm{~Hz})$ confirman la presencia de dos sustituyentes hidróxilos en las po- siciones 5 y 7, debido a la biogénesis de estos compuestos. En RMN- ${ }^{13} \mathrm{C}$ (Tabla 4) se observan seis señales que pertenecen a carbonos $\mathrm{sp}^{2}$ hidrogenados, una señal corresponde a un carbonilo, insaturado $(175,87 \mathrm{ppm})$ y ocho a carbonos $\mathrm{sp}^{2}$ cuaternarios. La información de los espectros coincide con los datos reportados (Mabry y Harborne 1982) para el kaempferol. 
Tabla 4. Desplazamientos químicos ( $\delta$ ) en el espectro $\mathrm{RMN}-{ }^{13} \mathrm{C}$ (DMSO- $\mathrm{D}_{6}, 100 \mathrm{MHz}$ ) de kaempferol [2].

\begin{tabular}{rrrrrr}
\hline $\mathrm{C}$ & $\mathrm{C}-2$ & $\mathrm{C}-3$ & $\mathrm{C}-4$ & $\mathrm{C}-5$ & $\mathrm{C}-6$ \\
$\delta$ & 146,79 & 135,61 & 175,87 & 160,67 & 98,16 \\
Tipo & $=\mathrm{C}-\mathrm{O}$ & $=\mathrm{C}-\mathrm{OH}$ & $>\mathrm{C}=\mathrm{O}$ & $=\mathrm{C}-\mathrm{OH}$ & $=\mathrm{C}-\mathrm{H}^{\prime}$ \\
$\mathrm{C}$ & $\mathrm{C}-7$ & $\mathrm{C}-8$ & $\mathrm{C}-9$ & $\mathrm{C}-10$ & $\mathrm{C}-1^{\prime}$ \\
$\delta$ & 163,84 & 93,43 & 156,09 & 103,01 & 121,91 \\
Tipo & $=\mathrm{C}-\mathrm{OH}$ & $=\mathrm{C}-\mathrm{H}$ & $=\mathrm{C}-\mathrm{O}$ & $=\mathrm{C}<$ & $=\mathrm{C}<$ \\
$\mathrm{C}$ & $\mathrm{C}-2^{\prime}$ & $\mathrm{C}-3^{\prime}$ & $\mathrm{C}-4^{\prime}$ & $\mathrm{C}-5^{\prime}$ & $\mathrm{C}-6^{\prime}$ \\
$\delta$ & 129,45 & 115,39 & 147,75 & 115,39 & 129,45 \\
Tipo & $=\mathrm{C}-\mathrm{H}$ & $=\mathrm{C}-\mathrm{H}$ & $=\mathrm{C}-\mathrm{OH}$ & $=\mathrm{C}-\mathrm{H}$ & $=\mathrm{C}-\mathrm{H}^{\prime}$ \\
\hline
\end{tabular}

\section{Conclusiones}

Los flavonoides son compuestos naturales que ya han sido descritos en algunos géneros de la familia Melastomataceas; sin embargo, en el género Aciotis no hay reportes previos de estos flavonoides.

Las partes aéreas de Aciotis polystachya (Bonplan) Triana (Melastomataceae), resultaron ser una fuente importante de flavonoides.

El análisis mediante técnicas de resonancia magnética nuclear $\left({ }^{1} \mathrm{H},{ }^{1} \mathrm{H}-\mathrm{COSY}\right.$ y HMQC $)$ de los compuestos [1] y [2] permitió la asignación, sin ambigüedades, de todas las señales en sus espectros de $\mathrm{RMN}-{ }^{1} \mathrm{H}$ y $\mathrm{RMN}-{ }^{13} \mathrm{C}$.

\section{Literatura citada}

Adriano AM, Rodríguez KV, Hiruma-Lima CA, Machado LR, Monteiro AR, Wagner V. 2006. Effect of Mouriri pusa extract on experimentally induced gastric lesions in rodents: Role of endogenous sulfhydryls compounds and nitric oxide in gastroprotection. J Etnhopharmol. 107: 431-41.

Bomfim-Patricio MC, Salatino A, Martins AB, Wurdack JJ, Salatino MLF. 2001. Flavonoids of Lavoisiera, Microlicia and Trembleya (Melastomataceae) and their taxonomic meaning. Biochem Syst Ecol. 29: 711-26.

Clausing G, Renner SS. 2001. Molecular and phylogenetics of Melastomataceae and Memecylaceae: Implications for characters evolution. Ann J Bot. 88: 486-98.

Cuesta-Sánchez JJ, Palacios-Duque L. 2007. Revisión del género Aciotis (Melastomataceae) en el departamento del Chocó, Colombia. (Trabajo de Grado). Quibdó: Facultad de Ciencias Básicas, Universidad Tecnológica del Chocó. $60 \mathrm{pp}$.

Freiré-Fierro A. 2002. Monograph of Aciotis (Melastomataceae). Syst Bot Monog. 62: 1-99.

García H. 1992. Flora medicinal colombiana. Bot Med. 2. Bogotá: Tercer Mundo Editores.

Isaza JH, Hidelyuki I, Takashi Y. 2001. A flavonol glycosidelignan ester and accompanying acylated glycosides from Monochaetum multiflorum. Phytochemistry. 58: 321-7.

Isaza JH, Jiménez F, Usma JLG, Restrepo JC. 2007. Actividad alelopática de algunas especies de los géneros de Miconia, Tibouchina, Henriettella, Tocota, Aciotis y Bellucia (Melastomataceae). Sci Techn. 1 (33): 409-13.

Li XC, Jacob MR, Pasco DS, Elsohly HN, Nimrod AC, Walker LA. 2001. Phenolic compounds from Miconia myriantha inhibiting Candida aspartic proteases. J Nat Prod. 64: 1282-5.

Mabry TJ, Harborne JB. 1982. The flavonoids: Advances in research. London, New York: Chapman and Hall Ltd. pp. 49-125.

Perry LM, Metzger J. 1980. Medicinal plants of east southeas of Asia. Cambridge: MIT Press.

Renner SS.1990. A revision of Rychanthera (Melastomataceae). Nordic J Bot. 9: 601-30.

Rodrigues J, Rinaldo D, Santos LCD, Vilegas W. 2007. An unusual C6-C6" linked flavonoid from Miconia cabucu (Melastomataceae). Phytochemistry. 68: 1781-4.

Salas-Moreno MH. 2011. Saponinas esteroidales en Aciotis polystachya (Bonplan) Triana (Melastomataceae). Revista Universidad Tecnológica del Chocó Institucional Investigación, Biodiversidad y Desarrollo. 30 (2): 15464.

Yoshida T, Ito H, Isaza JH. 2005. Pentameric ellagitannin oligomers in Melastomataceous plant-chemotaxonomic significance. Phytochemistry. 66: 1172-983.

Zhang Z, ElSohly HN, Li XC. 2003. Flavanone glycosides from Miconia trailii. J Nat Prod. 66: 39-41. 